\title{
Compact Microstrip Low-pass Filter with Wide Stop-band Using P-Shaped Resonator
}

\author{
Mohsen Hayati, Masoom Validi \\ Department of Electrical Engineering, Kermanshah Branch, Islamic Azad University, Kermanshah, Iran \\ Electrical Engineering Department, Faculty of Engineering, Razi University, Kermanshah-67149, Iran. \\ mohsen_hayati@yahoo.com,masoom_validi@yahoo.com \\ Farzin Shama, \\ Young Researchers and Elite Club, Kermanshah Branch, Islamic Azad University, Kermanshah, Iran. \\ f.shama@aut.ac.ir \\ Milad Ekhteraei \\ Young Researchers and Elite Club, Kermanshah Branch, Islamic Azad University, Kermanshah, Iran. \\ m.ekhteraei.ir@ieee.org
}

\begin{abstract}
This paper, a compact low-pass filter (LPF) with an Elliptic function response using P-shaped resonators is presented. The LPF with simple structure results in the low insertion loss less than $0.1 \mathrm{~dB}$ and high return loss better than $20 \mathrm{~dB}$, in the passband. The proposed filter with a $-3 \mathrm{~dB}$ cutoff frequency of 2.61 GHz is fabricated and measured. The measured results show that the proposed LPF has significant advantages such as a wide stopband from $2.9 \mathrm{GHz}$ to $17.3 \mathrm{GHz}$ with the suppression level better than $\mathbf{- 2 0} \mathbf{~ d B}$, and it has an acceptable sharp frequency response in the transition band. The measurement results are in good agreement with the simulation results.
\end{abstract}

Keywords — Low Pass Filter (LPF), Microstrip, P Shaped, Stop-band

\section{INTRODUCTION}

Microwave low-pass filters are the important blocks in modern wireless communication systems to eliminate unwanted signals [1]. Compact microstrip low-pass filters with wide stop-band, sharp roll off, high suppression in the stop-band and low insertion loss are widely demanded in microwave technologies. In [1], a LPF using radial stubs is presented, which provides an ultra wide stop-band; however, has a very gradual transition band. A LPF with low insertion and wide stop-band using modified semi-Elliptic and semi-circular microstrip patch resonator is presented in [2]. This filter has a low return loss in the pass-band and a large circuit size. In [3], a low-pass filter using coupled-line hairpin unit, semi-circle defected ground structures and semi-circle stepped-impedance shunt stubs is fabricated that has a sharp roll off, but it has narrow stop-band with $-20 \mathrm{~dB}$ rejection and low return loss in the pass-band. Also, it does not have a small size. A quasi Elliptic low-pass filter using symmetric rectangular coupled capacitors is presented in [4], which provides compact size and good return loss in the pass-band. But the rejection region is narrow; in addition, the transition band is gradual. In [5], a LPF using coupled-line hairpin units is presented. The filter does not have wide 
stop-band and sharp roll-off.

In this paper, a compact microstrip low-pass filter with a wide stop-band using P-shaped resonators is presented that result in low insertion loss and high return loss in the pass-band. The proposed filter has simple shape and achieves good performance in the stop-band region, pass-band and transition band.

\section{FILTER DESIGN}

The design process of the proposed filter is as follows:

i- Design of an Elliptic function resonator to achieve sharp cutoff response.

ii- Provide an LC model of the proposed P-shaped resonator in order to optimize the resonator.

iii- Design of the proposed filter that include the combination of the proposed resonator with suppressing cells.

\section{A. Design of Elliptic Function Resonator}

A prototype Elliptic-function low-pass resonator with desired cutoff frequency is selected, and the values of the circuit's elements are calculated [6], as shown in Figure 1. The characteristic impedances for microstrip lines with width 0.2 and 0.3 and $0.8 \mathrm{~mm}$ are $136 \Omega$ and $117 \Omega$ and $74 \Omega$, respectively.

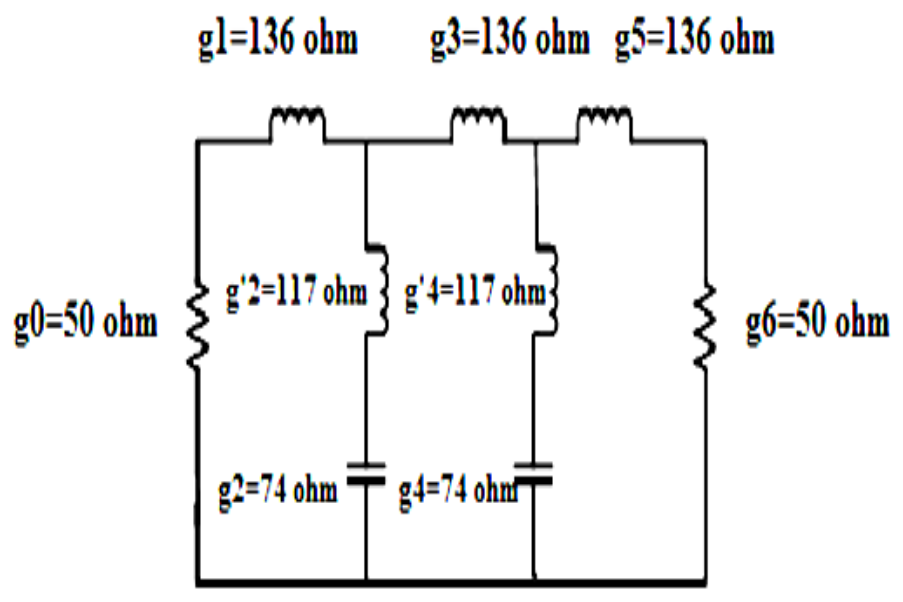

Fig. 1. The LC model of prototype Elliptic function low-pass resonator

To realize the Elliptic resonator, a P-shaped microstrip layout is proposed, as shown in Figure 2. In this layout, to realize the Elliptic function LC circuit, the high impedance lines with width of W1 and W2 are equivalent to inductance and the semicircle open-stubs are equivalent to capacitor. The coupling capacitance, between the semicircle open-end stubs, is ignored in this realization because the gap distance is large. 


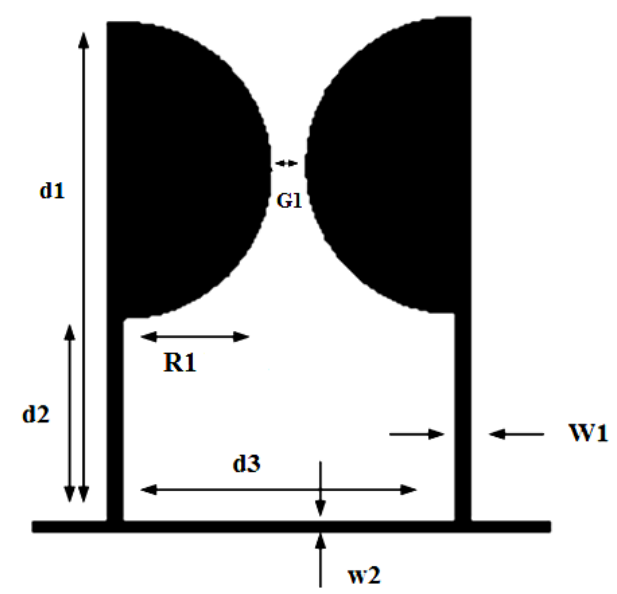

Fig. 2. The proposed resonator

The S-parameters of the P-shaped resonator are compared with the LC equivalent circuit frequency response, as shown in Figure 3. It can be seen clearly that the cutoff frequency of the proposed resonator is adjusted to $2.6 \mathrm{GHz}$ with an Elliptic response.

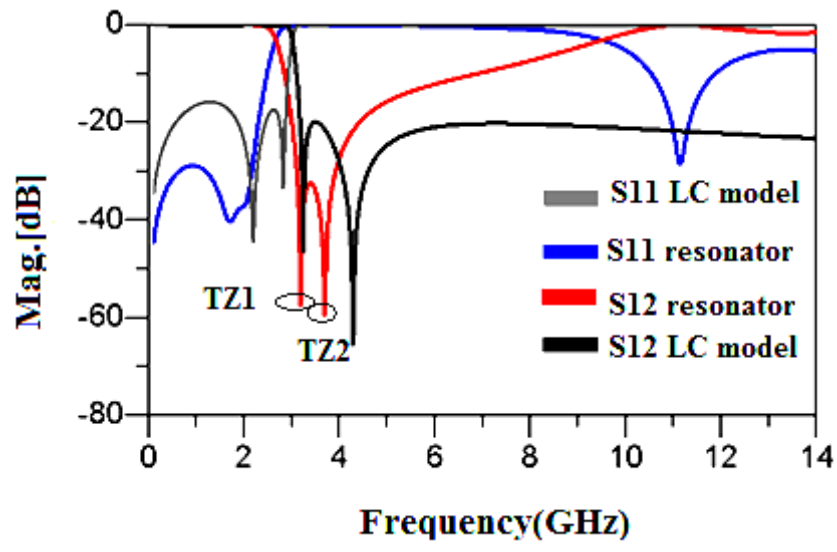

Fig. 3. The frequency response of the prototype filter

The resonator creates two transmission zeros at $3.3 \mathrm{GHz}$ and $3.9 \mathrm{GHz}$ with corresponding attenuation levels of $-55 \mathrm{~dB}$ and $-54 \mathrm{~dB}$, respectively, where return loss in the pass-band is $30 \mathrm{~dB}$. By increasing the width of $\mathrm{W} 1$ from $0.3 \mathrm{~mm}$ to $0.7 \mathrm{~mm}$ as shown in Figure 4 (a), decreasing $\mathrm{d} 2$ from 4 $\mathrm{mm}$ to $2 \mathrm{~mm}$ as shown in Figure 4 (b) and decreasing R1 from $2.5 \mathrm{~mm}$ to $1.5 \mathrm{~mm}$ as shown in Figure 4 (c), the transmission zeros move away from the lower frequencies. Therefore, the location of the transmission zeros can be controlled by tuning the values of $\mathrm{W} 1, \mathrm{~d} 2$ and $\mathrm{R} 1$.

With the increment of $\mathrm{d} 3$ from $6 \mathrm{~mm}$ to $6.5 \mathrm{~mm}$ with a step of $0.25 \mathrm{~mm}$, the attenuation level in the stop-band region is increased from $3.2 \mathrm{GHz}$ to $4 \mathrm{GHz}$. Also, the effect of increment of G1 is similar to the effect of the increment of $\mathrm{d} 3$. The effect of variation of $\mathrm{d} 3$ and $\mathrm{G} 1$ on the frequency response of the resonator is shown in Figure 4(d). But with increment of w2 from $0.2 \mathrm{~mm}$ to $0.6 \mathrm{~mm}$ with step of $0.2 \mathrm{~mm}$, the attenuation level in the stop-band region is decreased, as shown in Figure 4(e). Therefore, the dimensions of these parameters are optimized using optimization tools in the ADS software, which uses the local gradient optimization process. 


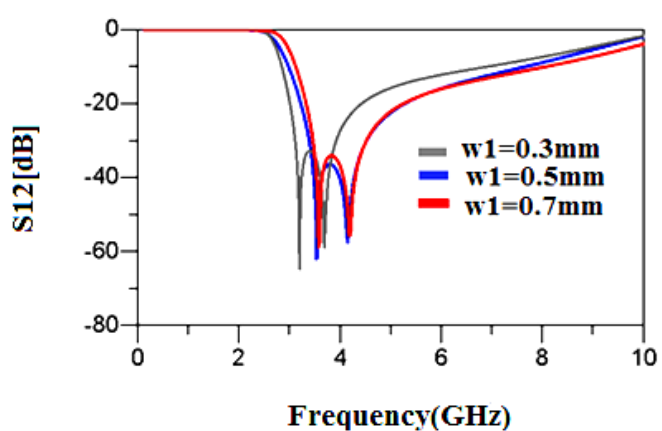

(a)

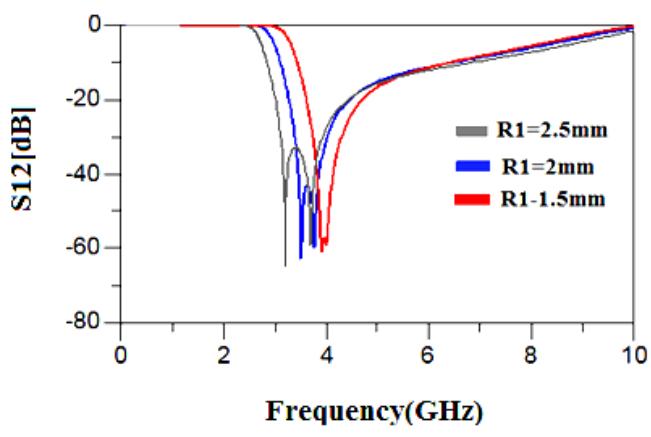

(c)

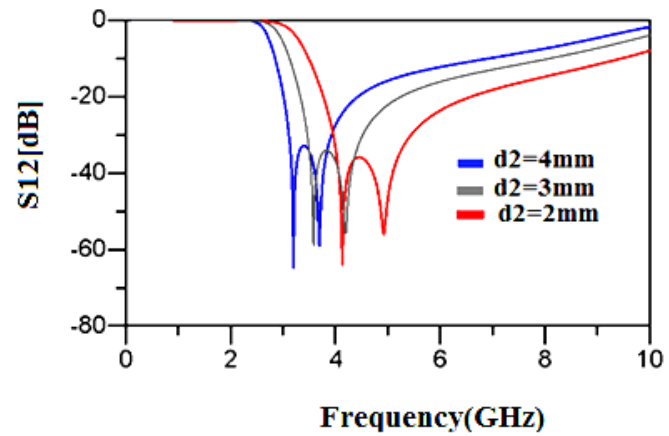

(b)

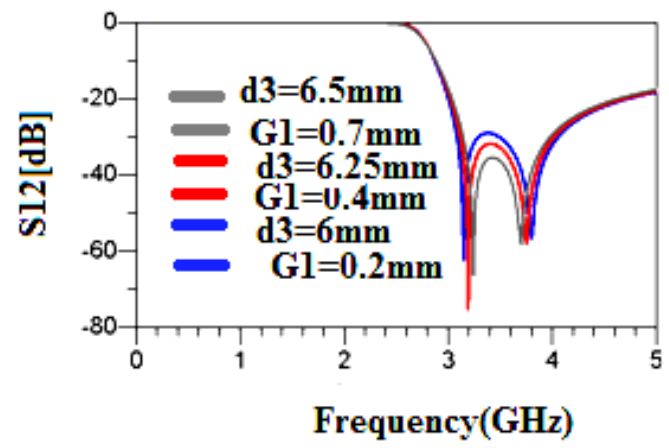

(d)

Fig. 4. (a) The S12 parameter of the proposed resonator as a function of W1 (b) The S12 parameter of the proposed resonator as a function of $\mathrm{d} 2$ (c) The S12 parameter of the

Proposed resonator as a function of R1 (d) The S12 parameter of the proposed resonator as a function ofd3 and G1 (e) The S12 parameter of the proposed resonator as a function of W2

\section{B. The LC model of the proposed P-shaped resonator}

The accurate LC model of the proposed P-shaped resonator is shown in Figure 5. As observed, Cg1 is the coupling capacitance between the semicircle open-end stubs, L1 and L2 represent inductances of the main transmission line between input and output ports, L3 represents inductance of the stubs, $\mathrm{Cr}$ is the capacitance of the radial open-end stubs with respect to the ground.

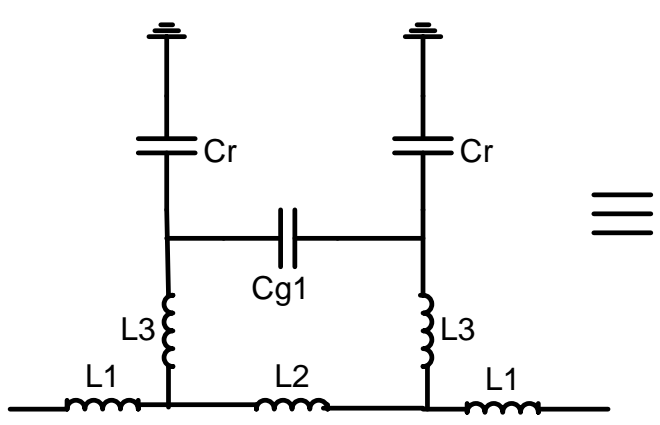

Fig. 5. The LC Model of the proposed resonator. 
By considering the open-end radial stubs as a unit, which is shown in Figure 6; the capacitance of the radial stub can be obtained through one port -parameter using (1) and (2) [7].

$$
C=\frac{-1}{2 \pi f\left(i m a g\left(Z_{\text {in }}\right)\right)}
$$

where Zin can be calculated as:

$$
Z_{\text {in }}=50\left[\frac{\left(1+S_{11}\right)}{\left(1-S_{11}\right)}\right]
$$

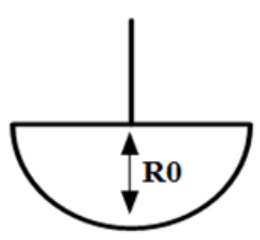

(a)

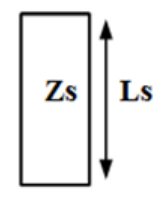

(b)

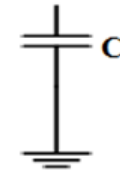

(c)

Fig. 6.The configuration of the capacitive elements. (a)Radial stub. (b)Straight stub. (c)The equivalent circuit of (a) and (b).

For the straight stub, the equivalent capacitance can be directly obtained as (3) when the effective electrical length is less than a quarter-wave length [7]:

$$
C=\frac{\tan \left(\beta L_{s}\right)}{2 \pi f Z_{s}}
$$

where $\beta$, Ls and Zs represent the propagation constant, length of the straight stub and impedance of the straight stub, respectively. The other parameters of the accurate LC model of the proposed resonator are obtained from [6]:

$$
\begin{aligned}
L_{i} & =\frac{Z_{0}}{g_{0}} \frac{g_{i}}{2 \pi f_{c}} \\
C_{i} & =\frac{g_{0}}{Z_{0}} \frac{g_{i}}{2 \pi f_{c}}
\end{aligned}
$$

where, $\mathrm{Li}$ and $\mathrm{Ci}$ represent the inductance and capacitance of the transmission line, respectively, Z0 represent source impedance, fc is cutoff frequency and $\mathrm{g} 0$ and gi represent either the inductance of a series inductor or the capacitance of a shunt capacitor.

Therefore, in the first step, the LC model of the elliptic function resonator with desired characteristics is designed. The parameters of the LC model of the proposed resonator are obtained from Equations (4) and (5). Then after obtaining LC values, in order to improve the accuracy of the response, the LC values are optimized by tuning tool in the ADS software, which uses the local gradient optimization process. The LC value of the proposed LPF is shown in Table.1.

The optimized dimensions of the proposed P-shaped layout are: $\mathrm{d} 1=10 \mathrm{~mm}, \mathrm{~W} 1=0.3 \mathrm{~mm}, \mathrm{~d} 3=6.5$ $\mathrm{mm}, \mathrm{W} 2=0.2 \mathrm{~mm}, \mathrm{~W} 3=0.8 \mathrm{~mm}, \mathrm{R} 1=2.2 \mathrm{~mm}, \mathrm{G} 1=0.7 \mathrm{~mm}$. 
Journal of Microwaves, Optoelectronics and Electromagnetic Applications, Vol. 15, No. 4, December 2016314 DOI: http://dx.doi.org/10.1590/2179-10742016v15i4633

TABle I. LC VALues of The Proposed Filter.

\begin{tabular}{|c|c|c|c|c|}
\hline Elements & L1(nH) & L2(nH) & $\mathbf{L 3}(\mathbf{n H})$ & $\mathbf{C r}(\mathbf{p F})$ \\
\hline $\begin{array}{c}\text { Calculated } \\
\text { L-C values }\end{array}$ & 0.82 & 5.9 & 3.43 & 0.17 \\
\hline $\begin{array}{c}\text { Optimized } \\
\text { L-C values }\end{array}$ & 0.82 & 5.11 & 3.23 & 0.13 \\
\hline
\end{tabular}

The physical lengths of the high- and low-impedance lines may be found by [6]:

$$
\begin{gathered}
l_{L i}=\frac{\lambda_{g L i}}{2 \pi} \sin ^{-1}\left(\frac{\omega_{c} L_{i}}{Z_{0 L i}}\right) \\
l_{C i}=\frac{\lambda_{g C i}}{2 \pi} \sin ^{-1}\left(\omega_{c} C_{i} Z_{0 c i}\right)
\end{gathered}
$$

where, $l_{\mathrm{Li}}$ and $\mathrm{l}_{\mathrm{ci}}$ represent the dimension's inductance and capacitance of the transmission line, $\mathrm{Z}_{0}$ represent source impedance, $\omega \mathrm{c}$ is cutoff frequency, $\lambda_{\mathrm{gL}}$ and $\lambda_{\mathrm{gc}}$ represent guided wavelengths $(\mathrm{mm})$ for inductance and capacitance, respectively.

\section{Design of the Proposed Filter}

To reach wide stop-band with good suppression of the harmonics in the stop-band region, a multiple resonator with different dimensions is used. In first step, two P-shaped units are added to the proposed resonator, as shown in Figure 7 (a). Figure 7(b) shows that the resonator 2 creates TZ3 and TZ4 in the stop-band region. On the other hand the stop bandwidth with $-17 \mathrm{~dB}$ attenuation level up to $9 \mathrm{GHz}$ is created.

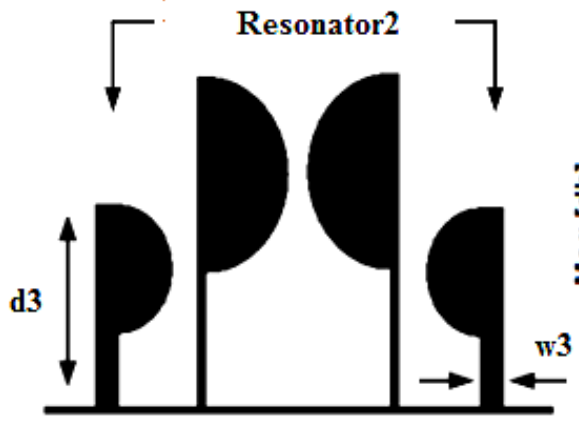

(a)

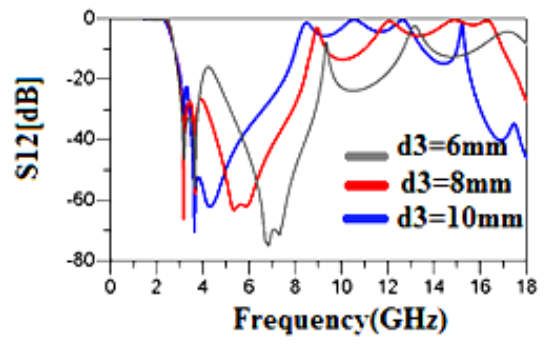

(c)

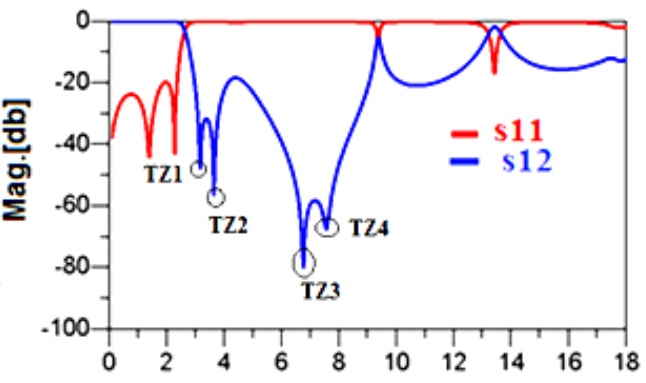

Frequency(GHz)

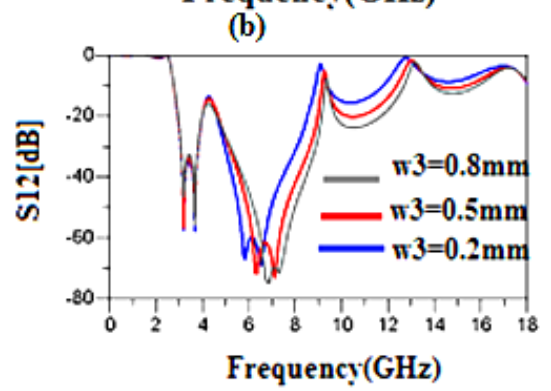

(d)

Fig. 7. (a) The proposed cascaded P-shaped resonator. (b) The simulated s-parameters of proposed cascaded P-shaped resonator. (c) The S12 parameter of the proposed resonator as a function of $\mathrm{d} 3$ (d) The S12 parameter of the proposed resonator as a function of $\mathrm{w} 3$ 
As shown in Figure 7(c), by increasing d 3 from $6 \mathrm{~mm}$ to $10 \mathrm{~mm}$, the transmission zeros are moved to the lower frequency (with considering the proposed LC model in Figure 5, by increasing d3, the capacitance of $\mathrm{Cr}$ and inductance of $\mathrm{L} 3$ are increased). When w3 is decreased from $0.8 \mathrm{~mm}$ to $0.2 \mathrm{~mm}$ as shown in Figure 7(d), the transmission zeroes are moved to the lower frequency (with considering the proposed LC model in Figure 5, by decreasing w3, the inductance of L3 is increased). The proposed structure does not have wide stop-band. To obtain a LPF with better stop bandwidth and attenuation level, third resonator is added to the structure of Figure 7(a). Each of P-shaped units adds a transmission zero, at about $11 \mathrm{GHz}$ that can guarantee the harmonics rejection in higher frequencies than $9 \mathrm{GHz}$, as shown in Figure 8. The layout of the proposed LPF consists of six P-shaped units, as illustrated in Figure 9 (a). Although the new added P-shaped units improve the stop-band region characteristics significantly, but it can create a more insertion loss in the pass-band imperceptibly. The structure parameters of the filter are: $\mathrm{Wf}=1.5 \mathrm{~mm}, \mathrm{df}=2 \mathrm{~mm}, \mathrm{~W} 1=0.2 \mathrm{~mm}, \mathrm{~d} 1=22 \mathrm{~mm}, \mathrm{~W} 2=0.3$ $\mathrm{mm}, \mathrm{d} 2=10 \mathrm{~mm}, \mathrm{~W} 3=\mathrm{W} 4=0.8 \mathrm{~mm}, \mathrm{~d} 3=6 \mathrm{~mm}, \mathrm{~d} 4=4 \mathrm{~mm}, \mathrm{R} 1=2.5 \mathrm{~mm}, \mathrm{R} 2=1.5 \mathrm{~mm}, \mathrm{R} 3=1$ $\mathrm{mm}, \mathrm{G} 1=0.7 \mathrm{~mm}, \mathrm{G} 2=1 \mathrm{~mm}, \mathrm{G} 3=0.4 \mathrm{~mm}$.

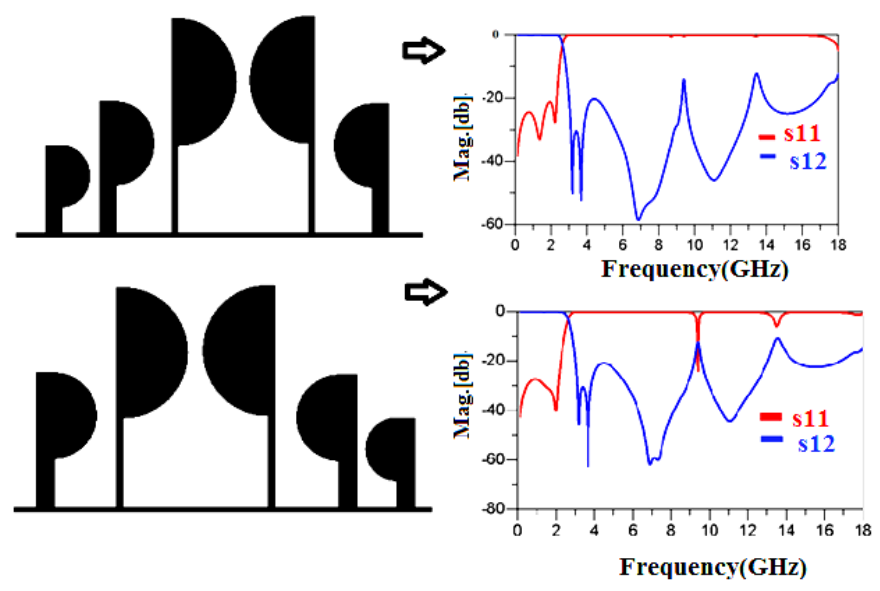

Fig. 8. The effect of adding a new P-shaped unit to the proposed cascaded P-shaped resonator.

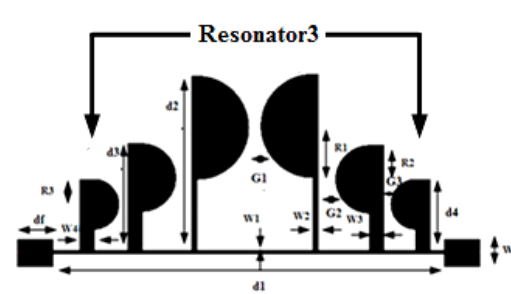

(a)

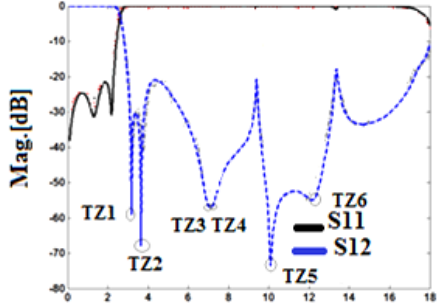

(b)

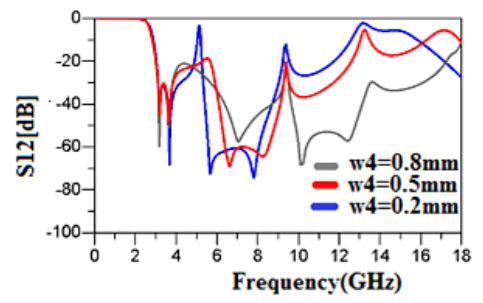

(c)

Fig. 9. (a) The proposed filter (b) The S12 parameter of the proposed filter (c) The S12 parameter of the proposed resonator as a function of 4 . 
As shown in Figure 9(c), when w4 is decreased from $0.8 \mathrm{~mm}$ to $0.2 \mathrm{~mm}$, the transmission zeroes are moved to the lower frequency. Therefore, the location of transmission zeros in the stop-band can be easily controlled by the variation of dimensions of the proposed filter parameters.

\section{MEASUREMENTS AND RESULTS}

The proposed LPF has been fabricated on a RT/Duroid 5880 substrate with a relative dielectric constant $\mathrm{\varepsilon r}=2.2$, thickness $\mathrm{h}=20 \mathrm{mil}$ and loss tangent of 0.0009 . The photograph of the fabricated filter is shown in Figure 10. The Simulations and measurements are performed using ADS software and HP 8757A network analyzer, respectively. The simulated and measured results are shown in Figure 11. A metallic cover is normally required for most microstrip circuit applications, such as filters. The presence of conducting top and side walls will affect both the characteristic impedance and the effective dielectric constant. In practice, a rule of thumb may be applied in the filter design to reduce the effect of cover: the height up to the cover should be more than 8 times and the distance to walls more than 5 times the substrate thickness. For more accurate design, the effect of cover, including the housing loss, can be taken into account by using full-wave EM simulation [6].

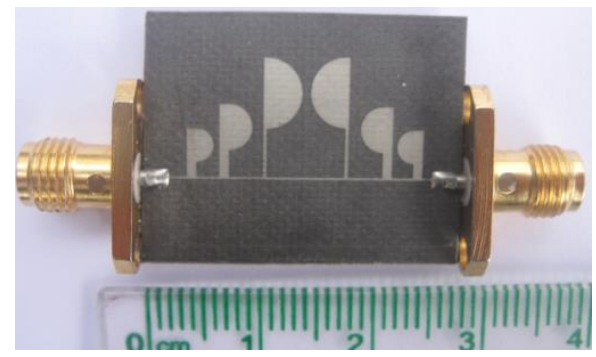

Fig. 10. The photograph of the fabricated LPF.
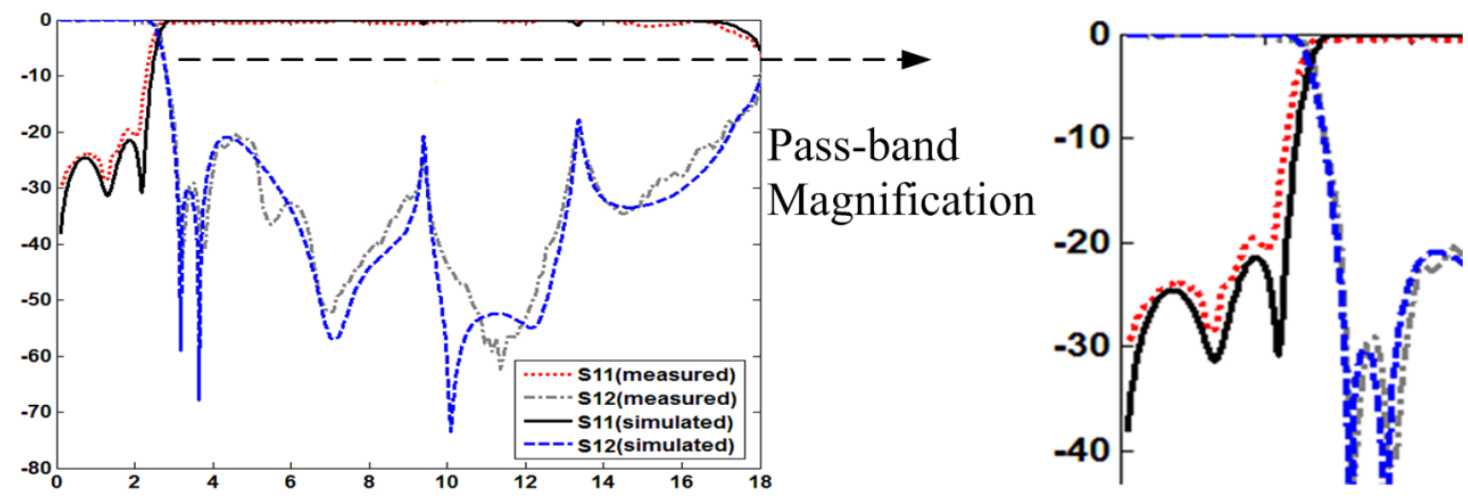

Fig. 11. The measured and simulated S-parameters of the fabricated LPF.

The filter has a $-3 \mathrm{~dB}$ cutoff frequency equal to $2.61 \mathrm{GHz}$ and insertion loss less than $0.1 \mathrm{~dB}$ in the pass-band from DC to $2 \mathrm{GHz}$. The return loss is better than $21 \mathrm{~dB}$ in the pass-band. The insertion loss has the suppression level better than $-20 \mathrm{~dB}$ from 2.9 up to $17.3 \mathrm{GHz}$ that obtains a wide stop-band. The return loss in the stop-band region is very close to $0 \mathrm{~dB}$. The size of the LPF is only $22 \times 10 \mathrm{~mm}^{2}$. Table 2 summarizes the performance of some other works. In this Table:

The roll off rate $\zeta$ is used to evaluate the roll off sharpness where a higher $\zeta$ represents a sharper roll 
off, which is defined as below [8]:

$$
\xi=\frac{\alpha_{\max }-\alpha_{\min }}{f_{S}-f_{C}}
$$

where $\alpha \max$ is the $-20 \mathrm{~dB}$ attenuation point; $\alpha$ min is the $-3 \mathrm{~dB}$ attenuation point; fs is the $-20 \mathrm{~dB}$ stop-band frequency; and fc is the $-3 \mathrm{~dB}$ cutoff frequency. The Relative stop-band bandwidth (RSB) is given by [8-9], where a higher RSB represents a wider stop-band region:

$$
R S B=\frac{\text { stopband }(-20 d B)}{\text { stopband center frequency }}
$$

The suppression factor (SF) is based on the stop-band suppression. A higher suppression degree in the stop-band leads to a greater SF. For instance, if the stop-band bandwidth is calculated under -20 $\mathrm{dB}$ restriction, then the SF is considered as 2. The normalized circuit size (NCS) is given by [8], which a lower NCS represents a smaller circuit size:

$$
N C S=\frac{\text { physical } \quad \text { size }(\text { lenght } \times \text { width })}{\lambda_{g}^{2}}
$$

This is applied to measure the degree of miniaturization of diverse filters, where $\lambda \mathrm{g}$ is the guided wavelength at $-3 \mathrm{~dB}$ cutoff frequency. The architecture factor (AF) can be recognized as the circuit complexity factor, which is signed as 1 when the design is $2 \mathrm{D}$ and as 2 when the design is $3 \mathrm{D}$. Finally, the figure of merit (FOM) is defined as below [8] and with the above discussion a higher FOM represents a better high-performance LPF:

$$
F O M=\frac{\zeta \times R S B \times S F}{N C S \times A F}
$$

TABLE II. PeRFoRMANCE COMPARISON With OTHER WORKS.

\begin{tabular}{|c|c|c|c|c|c|}
\hline Ref. & $\zeta$ & RSB & NCS & SF & FOM \\
\hline$[\mathbf{1}]$ & 5.28 & 1.66 & 0.01 & 2 & 1159 \\
\hline$[\mathbf{2}]$ & 30.35 & 1.42 & 0.061 & 2 & 1413 \\
\hline$[3]$ & 28.33 & 1.42 & 0.025 & 1 & 804 \\
\hline$[\mathbf{4}]$ & 37.8 & 1.16 & 0.02458 & 2 & 3576 \\
\hline$[\mathbf{5}]$ & 34 & 1.15 & 0.02072 & 1.7 & 3774 \\
\hline$[\mathbf{9}]$ & 29.3 & 1.49 & 0.1456 & 2 & 600 \\
\hline$[\mathbf{1 0}]$ & 52.85 & 1.22 & 0.0288 & 2 & 4478 \\
\hline$[\mathbf{1 1}]$ & 84 & 0.67 & 0.0857 & 2 & 985 \\
\hline$[\mathbf{1 2}]$ & 70 & 1.40 & 0.0272 & 1 & 1200 \\
\hline$[\mathbf{1 3}]$ & 16.9 & 1.38 & 0.0095 & 1.5 & 3682 \\
\hline This work & 47.22 & 1.48 & 0.020 & 2 & 6988 \\
\hline
\end{tabular}

As seen from the table, the proposed LPF exhibits highest figure of merit (6988) among the other 
Journal of Microwaves, Optoelectronics and Electromagnetic Applications, Vol. 15, No. 4, December 2016318 DOI: http://dx.doi.org/10.1590/2179-10742016v15i4633

published filters.

\section{CONCLUSION}

A compact microstrip low-pass filter using P-shaped resonators is proposed. This filter has a simple structure for easy fabrication. The proposed filter is simulated, fabricated and measured. Good agreement between measurement and simulation results is observed in both the pass-band and stopband regions. The measured results show that the proposed LPF has many satisfactory features such as compact size, low insertion loss, sharp roll off and wide stop-band in comparison with other works. The LPF with described performance can resolve the requirements in wireless communication systems and microwave applications.

\section{REFERENCES}

[1] Ma, K., Yeo, K.S. and Leoe, W.M.: Ultra-wide rejection band lowpass cell, Electronics Letters. 48, 99 -100 (2012).

[2] Hayati, M., Sheikhi, A. and Lotfi, A.: Compact lowpass filter with wide stopband using modified semi-elliptic and semi-circular microstrip patch resonator. Electronics letters. 46, 1507-1509 (2010).

[3] Wei, F., Chen, L., Shi, X.W., Huang, Q.L. and Wang, X.H.: Compact lowpass filter with wide stop-band using coupled-line hairpin unit. Electronics letters. 46, 88-90 (2010).

[4] Li, L. and Li, Z.F.: Compact quasi-elliptic lowpass filter using symmetric rectangular coupled capacitors. Electronics letters. 44, 124125 (2008)

[5] Sha, L., Lei, Z. and Sheng, S.: Stopband-Expanded Low-Pass Filters Using Microstrip Coupled-Line Hairpin Units. IEEE Microwave and wireless components letters. 18, 506-508 (2008).

[6] Hong, J.S. and Lancaster, M.J.: Microstrip Filters for RF/Microwave Applications, New York John Wiley \& Sons, Inc (2001).

[7] Ma, K. and Yeo, K., S.: New Ultra-Wide Stopband Low-Pass Filter Using Transformed Radial Stubs. IEEE transaction on microwave theory and techniques. 59, 604-611 (2011)

[8] Wang, J.P., Ge, L., Guo, Y. X. and Wu, W.: Miniaturized microstrip lowpass filter with broad stopband and sharp roll off. Electronics letters, 46, 573-575 (2010).

[9] Hayati, M. and Shama, F.: Compact Microstrip Low-Pass Filter with wide stopband using symmetrical U-shaped resonator. IEICE Electronics Express 9.3, 127-132 (2012).

[10] Hayati, M., \& Yousefzadeh, Y.: Compact Lowpass Filter with Wide Stopband Using Windmill-Shaped Resonator. Arabian Journal for Science and Engineering, 39(4), 3033-3038 (2014)

[11] Raphika, P. M., P. Abdulla, and P. M. Jasmine. : Compact lowpass filter with a sharp roll-off using patch resonators. Microwave and Optical Technology Letters 56.11, 2534-2536 (2014).

[12] Faraghi, Ahmad, Mohammad Ojaroudi, and Noradin Ghadimi. : Compact microstrip low-pass filter with sharp selection characteristics using triple novel defected structures for UWB applications. Microwave and Optical Technology Letters 56.4, 1007-1010 (2014).

[13] Chen, Xianhong, et al.: Compact lowpass filter with wide stopband bandwidth. Microwave and Optical Technology Letters 57.2, 367371 (2015). 